EMBRYARIDDLE
Aeronautical University

SCHOLARLY COMMONS

\section{International Journal of Aviation,} Aeronautics, and Aerospace

\title{
A thrust equation treats propellers and rotors as aerodynamic cycles and calculates their thrust without resorting to the blade element method
}

Phillip -. Burgers

Embry-Riddle Aeronautical University, burgersphil@gmail.com

Follow this and additional works at: https://commons.erau.edu/ijaaa

Part of the Aerodynamics and Fluid Mechanics Commons, Aeronautical Vehicles Commons, and the Propulsion and Power Commons

\section{Scholarly Commons Citation}

Burgers, P. -. (2019). A thrust equation treats propellers and rotors as aerodynamic cycles and calculates their thrust without resorting to the blade element method. International Journal of Aviation, Aeronautics, and Aerospace, 6(5). https://doi.org/10.15394/ijaaa.2019.1427

This Concept Paper is brought to you for free and open access by the Journals at Scholarly Commons. It has been accepted for inclusion in International Journal of Aviation, Aeronautics, and Aerospace by an authorized administrator of Scholarly Commons. For more information, please contact commons@erau.edu. 


\section{Introduction}

The equation for calculating the lift $L$ by a wing during the predesign stage is (Anderson, 2007)

$L=\frac{1}{2} \rho v_{\infty}^{2} C_{L} S_{r e f}$

Currently there is no equivalent "napkin-friendly" equation for calculating of the thrust $T$ of propellers and rotors as one must resort to labor-intensive computational algorithms like the vortex lattice method (VLM), the blade element method (BEM), or specialized CFD packages, tools that are indispensable during detailed design but time consuming during preliminary design. Two of the current legacy thrust equations for a rotor (Burgers, 2012) and a propeller (Burgers, 2016) lack the relevant physical parameters involved in the generation of thrust (i.e., $v_{\infty}$ and $\omega$ ) and use reference areas that are dimensionally proper but physically improper as they are not physically capable of exerting work onto the flow field during the generation of thrust, namely, the disk area $A$ of rotors, and $D^{2}$ of propellers:

$$
T_{\text {rotor }}=\rho v_{\text {tip }}{ }^{2} C_{T} A \quad T_{\text {propeller }}=\rho C_{T}\left(n^{2} D^{2}\right) D^{2}
$$

A third legacy equation for calculating the thrust $T$ of a rotor does account for relevant physical parameters (i.e., $v_{\infty}$ and $v_{t i p}$ ) and a physically proper reference area, the total blade area $S_{b}$ (Harris, 2011):

$T=\left(\frac{1}{4} v_{\infty}^{2}+\frac{1}{6} v_{t i p}^{2}\right) \rho C_{L} S_{b}$

This paper shows a slight difference between the above equation and the energy-based thrust equation derived in Section 5. This paper follows the current practice of naming both the forward force of a propeller and the upward vertical aerodynamic force generated by a rotor as thrust $T$. Transonic blade phenomena and blade kinematics during forward speeds (i.e., cyclic pitch) will not be addressed.

\section{Energy and Work of an Aerodynamic Cycle}

Both the propeller and rotor are assumed to operate as aerodynamic cycles with their available kinetic energy and work as input and output respectively, a treatment admittedly borrowed from thermodynamics. These concepts are covered next. 
Kinetic Energy: The dynamic pressure $q_{\infty}$, introduced by Prandtl in 1921, is the kinetic energy per unit volume of a fluid parcel as it translates at a velocity $v_{\infty}$ relative to a stationary lifting surface (Prandtl, 1921):

$q_{\infty}=\frac{1}{2} \rho v_{\infty}^{2}$

This term expression is reinterpreted as the specific kinetic energy $1 / 2 v_{\infty}^{2}$ of a lifting surface as it translates relative to a static fluid parcel of density $\rho$ placed at infinity. This reinterpretation is referenced as the kinematic pressure $Q_{\infty}$ :

$Q_{\infty}=\left(\frac{1}{2} v_{\infty}^{2}\right) \rho=e_{k \text { trans }} \rho$

When applied to a roto-translating propeller or rotor, the existing specific kinetic energy due to translation $e_{k \text { trans }}$ (per unit mass of the system) is algebraically added to its specific rotational kinetic energy $e_{k}$ rot (also per unit mass of the system):

$Q_{\infty}=\sum e_{k i} \rho=\left(e_{k \text { trans }}+e_{k r o t}\right) \rho=\left(\frac{1}{2} v_{\infty}^{2}+\frac{1}{2} \frac{I}{m} \omega^{2}\right) \rho$

The kinematic pressure $Q_{\infty}$ is the algebraic sum of the two sources of kinetic energies available at the roto-translating blades of propellers, and main and tail rotors, whereas the density paccounts for the physics of the surrounding flow field. For a propeller generating static thrust or a hovering rotor, both at $v_{\infty}=0$, the kinematic pressure $Q_{\infty}$ is:

$Q_{\infty}=\left(\frac{1}{2} \frac{I}{m} \omega^{2}\right) \rho=e_{k \operatorname{rot}} \rho$

Work: During the generation of thrust $T$, the propeller and rotor exert work $w$ onto the flow field by means of their reference area, the total blade area $S_{b}$. Throughout this paper, the reference area $S_{r e f}$ is defined as the summation of all physical aerodynamic surfaces as these (i) contribute to the generation of the aerodynamic force in an additive (wing of tail-configured airplane) or subtractive sense (i.e., its horizontal tail), (ii) while exerting work onto the flow field, and (iii) found (close to) perpendicular to the aerodynamic force, be it lift $L$, thrust $T$ or drag $D$. Note that this definition of $S_{\text {ref }}$ does not necessarily follow the legacy definition: the reference area $S_{r e f}$ of a wing is its planform area $S_{w}$; for a tail-configured 
airplane, its $S_{\text {ref }}$ is the sum of the wing and horizontal tail planform areas, $S_{p}$ $\left(=S_{w+h t}\right)$, and for propellers, main and tail rotors, it is their total blade area $S_{b}$. This definition of a reference area $S_{\text {ref }}$ does not necessarily negate other possible useful areas used in aerospace (i.e., the exclusive use of the wing area for a tail-configured aircraft or its wetted area), but their use when calculating figures of merit as $C_{L}$ or $C_{D}$ will be rendered limited as frequently, these will be able to be used to compare dissimilar systems nor be read on a stand-alone basis (Burgers, 2016).

The reference area $S_{r e f}$ is a relevant parameter in the calculation of the specific work $w$ during the generation of thrust $T$ :

$w=\frac{T}{\rho S_{b}}=\frac{[N][m]}{[\mathrm{kg}]}=\left[\frac{m^{2}}{s^{2}}\right]$

The blade loading $T / S_{b}$ of a propeller or rotor is an important parameter that can be easily varied during the pre-design stage to fine-tune their operating condition and prevent their stall in much the same way one estimates the wing area of a lifting surface $L / S_{\text {ref }}$ using Equation (1).

Aerodynamic Cycle: Throughout this paper, a propeller and rotor are assumed to operate as cycles with their specific kinetic energy $e_{k}$ as input and specific work $w$ exerted on the flow field as output:

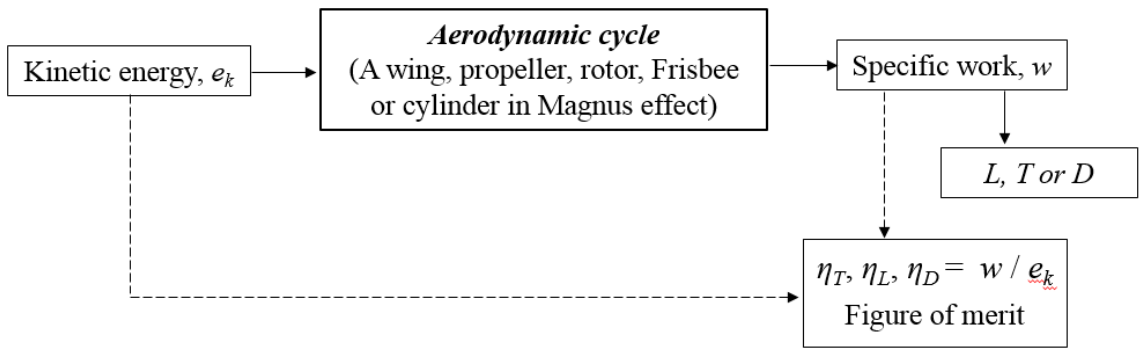

Figure 1. An aerodynamic system as a cycle and the corresponding figures of merit.

The ability of an aerodynamic cycle to generate thrust $T$ is quantified by the ratio of work $w$, and available kinetic energy $e_{k}$, a ratio referred to as the normalized thrust, $\eta_{T}$ :

$$
\eta_{T}=\frac{w}{\sum e_{k i}}=\frac{\frac{T}{\rho S_{b}}}{\frac{1}{2} v_{\infty}^{2}+\frac{1}{2} \frac{I}{m} \omega^{2}}
$$


The normalized thrust $\eta_{T}$ (as well as the accompanying normalized lift $\eta_{L}$ and drag $\eta_{D}$ ) can be derived by using the work-energy equation (Burgers \& Alexander, 2012) or the Buckingham $\pi$ theorem. Solving for thrust $T$, the thrust equation of a propeller and a rotor is:

$T=\left(\frac{1}{2} v_{\infty}^{2}+\frac{1}{2} \frac{I}{m} \omega^{2}\right) \rho \eta_{T} S_{b}$

For the case of $v_{\infty}=0$, this equation calculates the static thrust $T_{s t}$ of a propeller, or the thrust $T$ of a hovering main rotor in or out of ground effect, or the thrust of a static tail rotor. For the case of zero angular velocity, $\omega=0$, the above equation morphs into the ubiquitous lift equation of a translating lifting surface with its wing planform $S_{w}$ acting as a reference area, where the normalized lift $\eta_{L}$ equals the legacy lift coefficient $C_{L}$ (as both share the same definition of $S_{r e f}$ ). This equality is not valid for a tail-configured airplane, as its normalized lift $\eta_{L}$ uses $S_{\text {ref }}$ as the total planform area $S_{p}\left(=S_{w}+S_{h t}\right)$ whereas the lift coefficient $C_{L}$ uses $S_{r e f}=S_{w}$ (Burgers, 2016).

\section{Energy Sources}

Propellers and rotors generate thrust $T$ by converting an amount $\eta_{T}$ of their available kinetic energy $e_{k}$ into work $w$. According to this definition, Equation (9), a value of, say, $\eta_{T}=0.2$ should be interpreted as converting $20 \%$ of the kinetic energy $e_{k}$ available at the system to work $w$. Obviously, this rationale is inconvenient when the maximum value of the normalized thrust is found to be $\eta_{T}$ $\max >1$, which means there is more work $w$ exerted on the flow field than kinetic energy $e_{k}$ available at the system, a physical impossibility. A reason for this may be that the summation of energy available $\Sigma e_{k i}$ in the denominator of Equation (9) is limited for practical purposes to two sources: kinetic energy due to transition $e_{k}$ trans $\left(=1 / 2 v_{\infty}{ }^{2}\right)$, and rotation $e_{k r o t}\left(=1 / 2 I / m \omega^{2}\right)$, as these sources provide the bulk of the total energy available at the propeller and rotor.

Other kinematic and elastic energy sources relevant to a main rotor could be accounted for: the kinetic energy due to cyclic pitch, blade flapping, blade's lead-lag and the elastic energy contained within the blades due to bending, tension and torsion, these last three sources clearly not of a kinetic nature. By adding these terms, a more accurate thrust equation of a propeller and rotor is arrived at:

$$
\begin{aligned}
T=\left(\sum e_{k i}\right) \rho \eta_{L} S_{b} & =\left(\frac{1}{2} v_{\infty}^{2}+\frac{1}{2} \frac{I}{m} \omega^{2}+e_{k \text { cyc.pitch }}+e_{k \text { flap }}+e_{k \text { lead-lag }}+\right. \\
\left.e_{k \text { elastic }}\right) & \rho \eta_{L} S_{b}
\end{aligned}
$$


The addition of these supplemental sources contributes a negligible amount of energy. For example, the blade's kinetic energy due to the blade's cyclic pitch $e_{k}$ cyc.pitch about its longitudinal axis is analogous to the cyclic pitch of bird and insect flapping wings (i.e., causing the wing to pronate and supinate). The energy due to the flapping wing cyclic pitch has been shown to be negligible (Burgers, 2019).

Accounting for these supplemental energy sources may result in a maximum normalized thrust $\eta_{T \max }$ lower than 1, a statement that deserves more analysis (Moran \& Shapiro, 1988):

$\eta_{T \max }=\frac{w}{\sum e_{k}}=\frac{T_{\max }}{\left(\frac{1}{2} v_{\infty}^{2}+\frac{1}{6} v_{t g}{ }^{2}+e_{k c y c . p i t c h}+e_{k \text { flap }}+e_{k \text { lead-lag }}+e_{k \text { elastic }}\right) \rho s_{b}}<1 ?$

Making the normalized thrust $\eta_{T}$ behave similarly to the thermodynamic efficiency $\eta_{t h}$ is a desirable goal that comes at the cost of complicating the equation, as shown above. As a result, only two energy sources, $e_{k \text { trans }}$ and $e_{k}$ rot, are suggested for roto-translating systems, as shown in Equation (9). The consistent adoption of this two-source energy term for roto-translating systems does not affect the meaningful comparison of the normalized thrust, lift and drag of a wide variety of aerodynamic systems (e.g., propellers and rotors, flapping wings, cylinders in Magnus effect, Frisbees) as long as this rationale is adopted consistently. Of course, the normalized lift $\eta_{L}$ of a system that operates with a single source of energy $e_{k}$ trans (i.e., a translating wing) can be compared meaningfully with the normalized thrust $\eta_{T}$ of a system that operates with two sources of energy, $e_{k}$ trans and $e_{k}$ rot (i.e., a rototranslating main rotor of a helicopter).

\section{Blade Velocity}

The specific moment of inertia $I / m$ in equation (10) relates to each blade of radius $R$ of a rotor or propeller:

$\frac{I}{m}=\frac{I_{\text {single blade }}}{m_{\text {single blade }}}=\frac{k m_{\text {single blade }} R^{2}}{m_{\text {single blade }}}=k R^{2}=\frac{1}{3} R^{2}$

The constant $k$ characterizes the distribution of mass along the length of the blade, assumed here to be a rod of constant cross-section as it rotates about its end. The value of $k$ is $1 / 3$ (Halliday, 1970) and has no aerodynamic implications. Replacing $I / m$ from Equation (13) into equation (10) results in:

$$
T=\left(\frac{1}{2} v_{\infty}^{2}+\frac{1}{2} \frac{1}{3} R^{2} \omega^{2}\right) \rho \eta_{T} S_{b}=\frac{1}{2} v_{b}^{2} \rho \eta_{L} S_{b}
$$


Based on the above equation, the square of a roto-translating blade $v_{b}^{2}$ is defined as:

$v_{b}{ }^{2}=v_{\infty}^{2}+\left(\sqrt{\frac{1}{3}} R \omega\right)^{2}=v_{\infty}^{2}+0.577^{2} R^{2} \omega^{2}=v_{\infty}^{2}+\frac{1}{3} v_{t i p}{ }^{2}$

Replacing the product $R \omega$ by $v_{t i p}$, the velocity of the blade's tip, we obtain:

$$
T=\left(\frac{1}{2} v_{\infty}^{2}+\frac{1}{6} v_{t i p}^{2}\right) \rho \eta_{L} S_{b}
$$

Based on the above equation, the tangential velocity $v_{t g}$ of the blade is defined as $0.577 R v_{t i p}$, and acting at a radial blade station $r=0.577 R$.

Despite their scalar origin, the sum of the square velocities mimics the sum of perpendicular vectors $\bar{v}_{\infty}$ and $\bar{v}_{t i p}$, which is true for a propeller blade, but not for rotor blades at their azimuth positions $\psi=90^{\circ}$ and $\psi=270^{\circ}$ where these velocity vectors are collinear! This fact notwithstanding, these velocities quantify the blades' translational and rotational kinetic energies, and are not dependent on the blades' azimuth.

\section{Comparing the Legacy and the Energy-based Thrust Equations}

The definition of the energy-derived blade velocity squared $v_{b}^{2}$, Equation (15), is different than the legacy, vector-based definition of $v_{b}^{2}$, shown below as a function of the tangential velocity $v_{t i p}$, and the translational velocity $v_{\infty}$, a function of its azimuth angle $\psi$ (Harris, 2011):

$v_{b}^{2}=\left(v_{t i p}+v_{\infty} \sin \psi\right)^{2}$

In the above equation, the translation velocity $v_{\infty}$ is zero at the azimuth angles $\psi=0^{\circ}$ and $\psi=180^{\circ}$, and so, its average value over one blade revolution is expected to be somewhat smaller than the energy-based value of $v_{b}^{2}$ in Equation (15).

The legacy thrust equation of a rotor with $b$ blades, a blade airfoil with a lift slope $a_{\infty}$, a blade chord $c$, and an angle of attack $\theta$ uses expression (17) for $v_{b}{ }^{2}$. The product $b \cdot c \cdot R$ in the equation below equals the total blade area $S_{b}$, and the second product $a_{\infty} \cdot \theta$ is the lift coefficient $C_{L}$ of the blade (Harris, 2011). The resulting legacy thrust equation, obtained by BEM and same as equation (3), is repeated below:

$$
T=\frac{b \rho a_{\infty} c R \theta}{6}\left[\frac{3}{2} v_{\infty}^{2}+(\omega R)^{2}\right]=\left(\frac{1}{4} v_{\infty}^{2}+\frac{1}{6} v_{t i p}^{2}\right) \rho C_{L} S_{b}
$$


For ease of comparison, the energy-based thrust equation (16) is repeated below:

$T=\left(\frac{1}{2} v_{\infty}^{2}+\frac{1}{6} v_{t i p}^{2}\right) \rho \eta_{T} S_{b}$

Note the discrepancy in bold between the two equations: the presence of the $1 / 4$ in Equation (18) compared to the $1 / 2$ in Equation (19). This difference does not affect the calculation of static thrust by a propeller or thrust by a hovering rotor, as in both cases $v_{\infty}=0$, and as a result, the thrust equation is:

$T=\frac{1}{6} v_{t i p}^{2} \rho \eta_{T} S_{b}$

Next, the thrust $T$ of a single roto-translating blade (as $b=1, S_{\text {ref }}$ is the area of that single blade) is calculated next using the legacy equation (18) and the energy-based equation (19). The blade has a length $R$ of $9.14 \mathrm{~m}$. (30 ft.), a chord $c$ of $0.3 \mathrm{~m}$. (1 ft.), resulting in a reference area $S_{b}$ of $2.78 \mathrm{sq} . \mathrm{m}$. (30 sq. ft.), as it rotates at $300 \mathrm{rpm}$ (an angular velocity $\omega$ of $31.421 / \mathrm{s}$ ) and a forward speed $v_{\infty}$ that varies from hover to a forward speed of, say, $45.72 \mathrm{~m} / \mathrm{s}(150 \mathrm{ft} / \mathrm{s})$ while operating at a constant lift coefficient $C_{L}$ in Equation (18), and a constant normalized thrust $\eta_{T}$ in Equation (19) of 0.7, while operating at sea level $\left(\rho=1.225 \mathrm{~kg} / \mathrm{m}^{3}=0.002378\right.$ slugs $\left./ \mathrm{ft}^{3}\right)$. Results are shown in Figure 2 . 


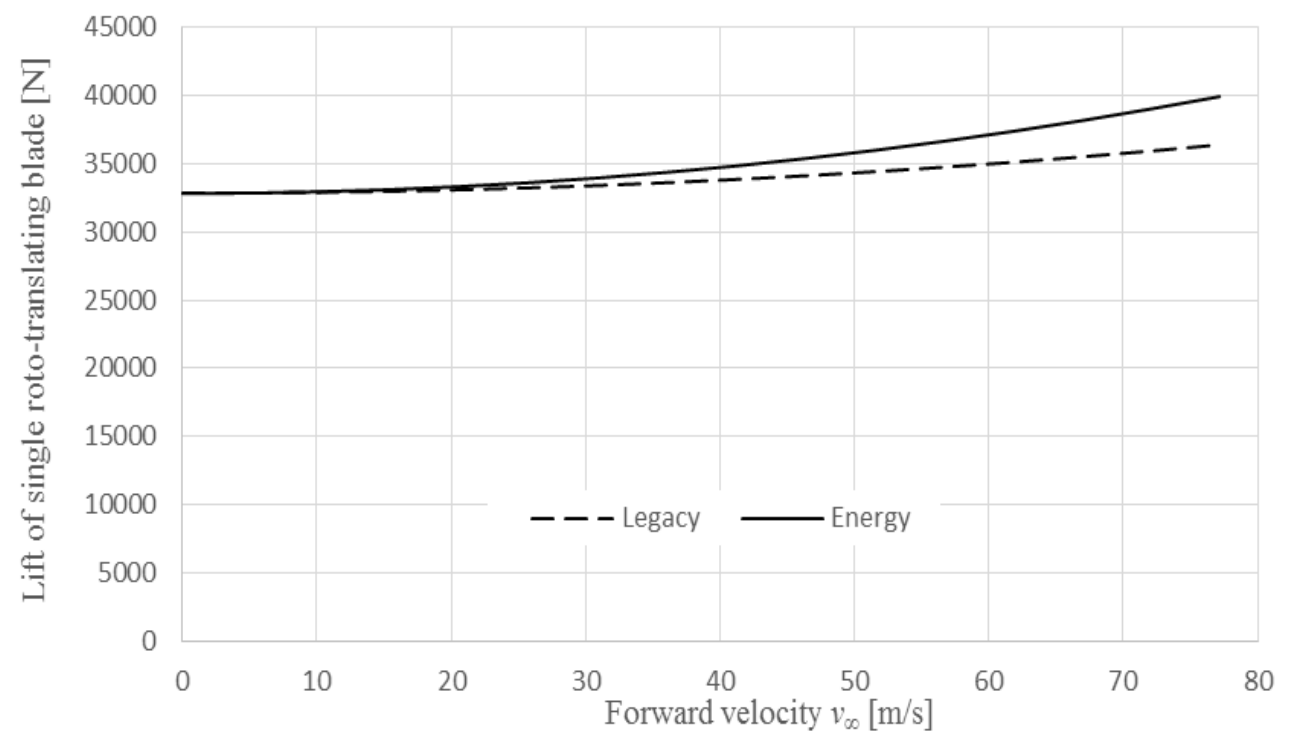

Figure 2. Comparison of legacy and energy-based thrust equation.

During hover, both equations yield the same result. At a forward speed of $30 \mathrm{~m} / \mathrm{s}(100 \mathrm{ft} / \mathrm{s})$, the energy-based equation yields a thrust value that is $2 \%$ higher, and at $75 \mathrm{~m} / \mathrm{s}(250 \mathrm{ft} / \mathrm{s}), 10 \%$ higher.

The lift coefficient $C_{L}$ in Equation (18) does not have a formal physicsbased definition and so, using the vector-based definition of the square of the velocity $v_{b}^{2}$, Equation (17), the numerical value of $C_{L}$ that is not restricted by a physical definition. This same rationale is also applicable when calculating the legacy thrust coefficient $C_{T}$ of a propeller using the square of its "velocity" $n^{2} D^{2}$, as the resulting value of $C_{T}$ is not expected to satisfy a physical definition. When using Equation (9), one arrives at a normalized thrust $\eta_{T}$ that is defined as the ratio of work and kinetic energy, $w / e_{k}$ and so, it is important to use the square of the velocity $v_{b}^{2}$ that is indicative of the amount of kinetic energy $e_{k}$ available at the propeller and rotor. The differences between these two numbers is that the normalized thrust can be used as a figure of merit with a physical meaning that allows for a meaningful comparison between dissimilar systems.

Advance Ratio and a Modifier

The advance ratio $J$ of a propeller or rotor of diameter $d$, translating at velocity $v_{\infty}$, and rotating at $n \mathrm{rpm}$ is (McCormick, 1979):

$$
J=\frac{v_{\infty}}{n d}
$$


Introducing the following geometric and kinematic-based equalities:

$$
R^{2}=\frac{d^{2}}{4} ; \quad \omega^{2}=4 \pi^{2} n^{2} ; \quad d^{2} n^{2}=\frac{v_{\infty}^{2}}{J^{2}}
$$

From the above equations, the square of the tangential velocity $R^{2} \omega^{2}$ equals:

$$
R^{2} \omega^{2}=\left(\frac{d^{2}}{4}\right)\left(4 \pi^{2} n^{2}\right)=\pi^{2} \frac{v_{\infty}^{2}}{J^{2}}
$$

Replacing $R^{2} \omega^{2}\left(=v_{t i p}{ }^{2}\right)$ by $\pi^{2} v_{\infty}{ }^{2} / J^{2}$ in equation (15) and moving the term $v_{\infty}{ }^{2}$ out of the parentheses as a common factor, we define the blade velocity $v_{b}$ as:

$$
v_{b}=\left(v_{\infty}^{2}+\frac{1}{3} v_{t i p}{ }^{2}\right)^{\frac{1}{2}}=\left(v_{\infty}^{2}+\frac{1}{3} R^{2} \omega^{2}\right)^{\frac{1}{2}}=v_{\infty}\left[1+\frac{1}{3}\left(\frac{\pi}{J}\right)^{2}\right]^{\frac{1}{2}}
$$

For lack of a better name, the term in brackets is referred to as the modifier as it modifies the blade's translation velocity $v_{\infty}$ to the roto-translating blade velocity $v_{b}$. The thrust of a propeller is written next as a function of the modifier:

$$
T=\frac{1}{2} v_{\infty}^{2}\left[1+\frac{1}{3}\left(\frac{\pi}{J}\right)^{2}\right] \rho \eta_{T} S_{b}=\frac{1}{2} v_{b}^{2} \rho \eta_{T} S_{b}=Q_{\infty} \eta_{T} S_{b}
$$

For a translating wing, $J=\infty$, and making $\pi / J=0$ and replacing $T$ by $L, \eta_{T}$ by $\eta_{L}$ and $S_{b}$ by the wing planform area $S_{w}$, the equation morphs to the lift equation of a lifting surface (with $S_{r e f}=S_{w}$ ) or that of a tail-configured aircraft (with $S_{r e f}=S_{w}$ $\left.+S_{h t}\right)$ (Burgers, 2016).

A more concise equation can be written by borrowing the definition of equivalent thrust area $f_{T}\left(=\eta_{T} S_{b}\right)$ from aircraft design (Roskam, 1983):

$T=Q_{\infty} f_{T}$

The drag $D$ of a roto-translating, windmilling propeller $\left(v_{\infty} \neq 0, \neq \omega\right)$ is:

$$
D=\frac{1}{2} v_{\infty}^{2}\left[1+\frac{1}{3}\left(\frac{\pi}{J}\right)^{2}\right] \rho \eta_{D} S_{b}=Q_{\infty} \eta_{D} S_{b}
$$

The drag $D$ of a static wind turbine (assuming wind speed $v_{\infty} \approx 0$ ), related to power extraction, is: 
$D=\frac{1}{6} v_{t i p}^{2} \rho \eta_{D} S_{b}$

Note that the thrust $T$ and lift $L$ and related normalized thrust $\eta_{T}$ and lift $\eta_{T}$ are evaluated when work $w$ is exerted on the flow field, whereas the drag $D$ and the normalized drag $\eta_{D}$ is evaluated when the flow field exerts work on the system.

The modifier also corrects the Reynolds number $R e_{\infty}$ of a propeller blade translating at a speed $v_{\infty}$ to its proper Reynolds number $R e_{b}$ by accounting for its actual blade speed $v_{b}$ :

$R e_{b}=\frac{\rho v_{b} c}{\mu}=\frac{\rho v_{\infty} c}{\mu}\left[1+\frac{1}{3}\left(\frac{\pi}{J}\right)^{2}\right]^{\frac{1}{2}}=R e_{\infty}\left[1+\frac{1}{3}\left(\frac{\pi}{J}\right)^{2}\right]^{\frac{1}{2}}$

The length of the chord $c$ is measured at a blade station $r=0.577 \cdot R$.

The following table reviews how translation-related parameters are modified to account for their rotation.

Table 1

Translation-related parameters modified to roto-translating parameters

\begin{tabular}{|c|c|c|c|c|}
\hline $\begin{array}{l}\text { Paramete } \\
r\end{array}$ & multiplied by & $\begin{array}{c}\text { converts } \\
\text { to }\end{array}$ & Equation (\#) & Observations \\
\hline$q_{\infty}$ & $1+1 / 3(\pi / J)^{2}$ & $Q_{\infty}$ & (25) & $\begin{array}{l}\text { Dynamic pressure to kinetic } \\
\text { pressure }\end{array}$ \\
\hline$v_{\infty}$ & $\begin{array}{l}{[1+1 / 3} \\
\left.(\pi / J)^{2}\right]^{1 / 2}\end{array}$ & $v_{b}$ & (24) & $\begin{array}{l}\text { Translation blade velocity to } \\
\text { roto-trans. blade velocity }\end{array}$ \\
\hline$e k_{\text {trans }}$ & $1+1 / 3(\pi / J)^{2}$ & $\begin{array}{c}e k_{\text {trans }}+ \\
e k_{\text {rot }}\end{array}$ & - & $\begin{array}{l}\mathrm{KE} \text { due to translation to } \mathrm{KE} \\
\text { due to roto-translation }\end{array}$ \\
\hline$R e_{\infty}$ & $\begin{array}{l}{[1+1 / 3} \\
\left.(\pi / J)^{2}\right]^{1 / 2}\end{array}$ & $R e_{b}$ & (29) & $\begin{array}{l}\text { Re of translating blade to } \mathrm{Re} \\
\text { roto-transl. blade }\end{array}$ \\
\hline$f_{\text {T prop }}$ & $1+1 / 3(\pi / J)^{2}$ & $f_{D a f}$ & (38) & $\begin{array}{l}\text { Equivalent thrust area to } \\
\text { equivalent drag area }\end{array}$ \\
\hline
\end{tabular}

The relationship between the equivalent thrust area $f_{T \text { prop }}$ of a propeller and the equivalent drag area $f_{D}$ af of an aircraft's airframe is shown in the above table.

\section{Numerical Characterization of a Propeller}

A series of small propellers where tested in the UIUC wind tunnel (Uhlig \& Selig, 2008). One particular propeller test showed a clear blade stall while operating at the highest possible blade angle pitch setting of $39^{\circ}$, measured at $75 \%$ radius: a two-bladed Ramoser varioPROP 9.9D of diameter $d$ of $25.14 \mathrm{~cm}$ (9.9”). 
This propeller has the following attributes: a blade length of $125.57 \mathrm{~mm}$ $(0.412 \mathrm{ft})$, a specific moment of inertia, $I / m$, of $5258.31 \mathrm{~mm}^{2}\left(0.0566 \mathrm{ft}^{2}\right)$, calculated using Equation (13). The area of a single blade $S_{l b}$, is $2461.93 \mathrm{~mm}^{2}\left(0.0265 \mathrm{ft}^{2}\right)$ is obtained from the blade's chord evolution along the blade, $r / R$ in (Uhlig \& Selig, 2008). The total blade area of the propeller, $S_{b}$, of $4942.44 \mathrm{~mm}^{2}\left(0.0532 \mathrm{ft}^{2}\right)$, is used as $S_{\text {ref }}$.

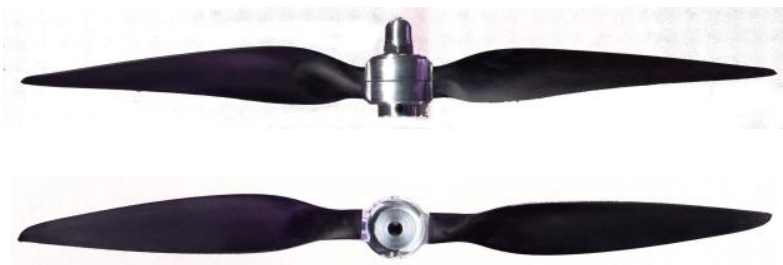

Side and planform views

Figure 3. Side and plan views of the Ramoser varioPROP 9.9D.

During this particular test, the propeller operated at $\approx 4000 \mathrm{rpm}$ and a blade pitch angle of $39^{\circ}$ as the incoming translation velocity in the wind tunnel, $v_{\infty}$, is varied from $0 \mathrm{~m} / \mathrm{s}$ to $24.61 \mathrm{~m} / \mathrm{s}(80.77 \mathrm{ft} / \mathrm{s})$. At an advance ratio $J\left(=v_{\infty} / n d\right)$ of zero, the propeller has zero forward speed, $v_{\infty}=0$, and the corresponding available specific kinetic energies are $e_{k}$ trans $=0 \mathrm{~m}^{2} / \mathrm{s}^{2}$, and $e_{k \text { rot }}=462.27 \mathrm{~m}^{2} / \mathrm{s}^{2}(4975.93$ $\mathrm{ft}^{2} / \mathrm{s}^{2}$ ) while generating a static thrust $T$ of $2.685 \mathrm{~N}(0.6037 \mathrm{lb}$.). At an advance ratio $J$ of 1.46 , its maximum translational velocity $v_{\infty}$ is $24.61 \mathrm{~m} / \mathrm{s}(80.77 \mathrm{ft} / \mathrm{s})$, and the available specific kinetic energies are $e_{k \text { trans }}=303 \mathrm{~m}^{2} / \mathrm{s}^{2}\left(3262 \mathrm{ft}^{2} / \mathrm{s}^{2}\right)$, and $e_{k}$ rot of $467.11 \mathrm{~m}^{2} / \mathrm{s}^{2}\left(5028\left(\mathrm{ft}^{2} / \mathrm{s}^{2}\right)\right.$ as the propeller generates a thrust $T$ of $-0.3 \mathrm{~N}(-0.068$ lb.).

A useful parameter from wing design is the blade loading $T / S_{b}$. At an advance ratio $J$ of 0.4 , the blades operate at a normalized thrust $\eta_{T}$ is 0.95 and the thrust $T$ is $2.79 \mathrm{~N}\left(0.28 \mathrm{~kg} / 0.628 \mathrm{lb}\right.$.). The resulting blade loading $T / S_{b}$ is $56.6 \mathrm{~kg} / \mathrm{m}^{2}$ $(11.8 \mathrm{lb} / \mathrm{sq}$. ft.), comparable to the wing loading of a sailplane.

The following figure shows the normalized thrust $\eta_{T}$ and the legacy thrust coefficient $C_{T}$ plotted against the advance ratio: 
$\eta_{T}$ and $C_{T} v s . J$

@ 4000 rpm / blade pitch=39 deg. @ 75\% R

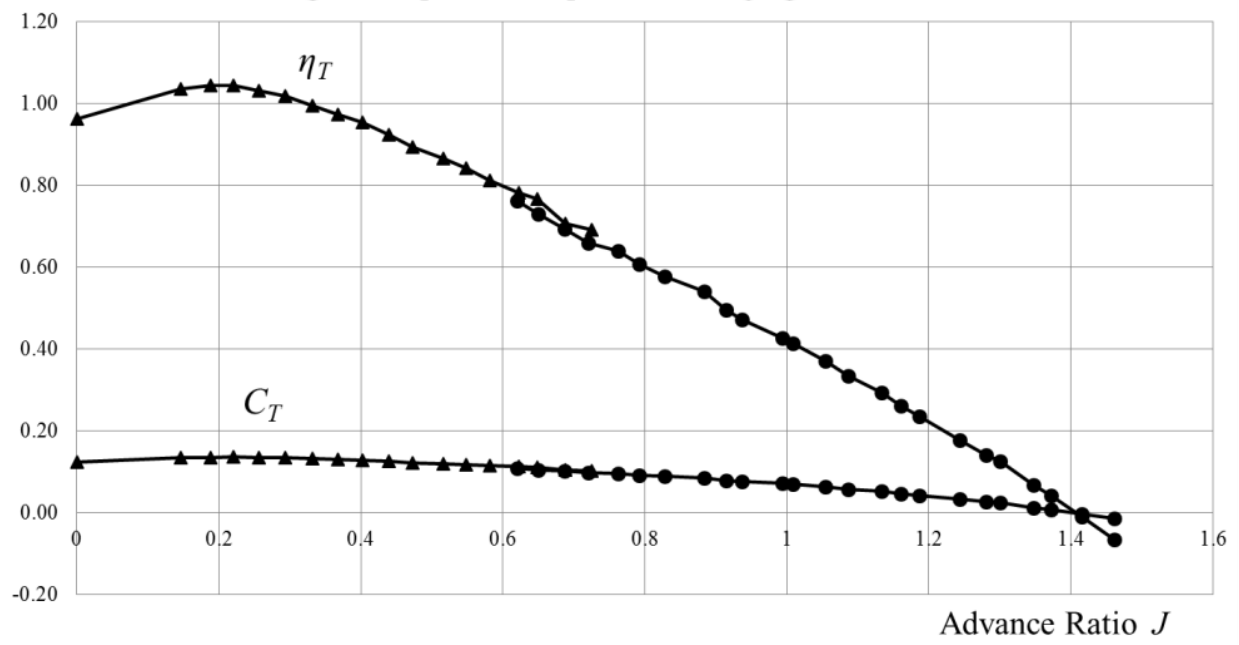

Figure 4. Normalized thrust and the legacy thrust coefficient against $J$ for a $39^{\circ}$ blade pitch.

The maximum normalized thrust $\eta_{T \max }$ and the thrust coefficient $C_{T \max }$ is the operating point at which the blades generate their maximum thrust, $T_{\max }$, of 2.95 $\mathrm{N}(0.663 \mathrm{lb}$.) at an advance ratio, $J$, of 0.188 , and a Reynolds number of $\approx 37,000$. Beyond this operating condition, the blades stall. The maximum normalized thrust, $\eta_{T \max }$ is 1.044 (equal to its $C_{L \max }$ ) is calculated by solving for $\eta_{T \max }$ in Equation (25):

$\eta_{T \max }=\frac{T_{\max }}{\frac{1}{2} \rho v_{b}^{2} S_{b}}=\frac{T_{\max }}{\frac{1}{2} \rho v_{\infty}^{2}\left[1+\frac{1}{3}\left(\frac{\pi}{J}\right)^{2}\right] S_{b}}=\frac{w_{\max }}{\mathrm{e}_{\text {k avail }}}=1.044$

Whereas $C_{T}$ lacks a physical interpretation, the $\eta_{T \max }$ is interpreted as the ratio of the maximum specific work $w$ exerted by the propeller onto the flow field per kinetic energy available at the propeller prior to stall.

Note that this maximum value exceeds 1 , and as discussed, comes close to conforming to the expected behavior from the efficiency $\eta_{t h}$ in thermodynamics. As mentioned, the inclusion of supplemental sources of kinetic and elastic sources of energy may correct this, at the expense of impracticality.

The following table compares the normalized thrust, $\eta_{T}$, the normalized torque, $\eta_{Q}$, the normalized power, $\eta_{P}$, and the propeller efficiency $\eta_{\text {prop }}$ with their analogous legacy equations: 
Table 2

Comparative table of legacy coefficients and normalized numbers for the thrust $T$, torque $Q$, power $P$ and efficiency $\eta_{\text {prop }}$ of a propeller

\begin{tabular}{cccc}
\hline \hline Parameter & $\begin{array}{c}\text { Legacy } \\
\text { Coefficient }\end{array}$ & Normalized Number & Observations \\
\hline Thrust & $C_{T}=\frac{T}{\rho n^{2} D^{4}}$ & $\eta_{T}=\frac{T}{\frac{1}{2} \rho v_{b}^{2} S_{b}}$ & $C_{T} \neq \eta_{T}$ \\
Torque & $C_{Q}=\frac{Q}{\rho n^{2} D^{5}} \quad \eta_{Q}=\frac{Q}{\frac{1}{2} \rho v_{b}^{2} S_{b} D}$ & $C_{Q} \neq \eta_{Q}$ \\
Power & $C_{P}=\frac{P}{\rho n^{3} D^{5}} \quad \eta_{P}=\frac{P}{\frac{1}{2} \rho v_{b}^{2} S_{b} n D}$ & $C_{P} \neq \eta_{P}$ \\
Efficiency & $\eta_{p r o p}=\frac{T v_{\infty}}{P}=\frac{C_{T}}{C_{P}} J=\frac{\eta_{T}}{\eta_{P}} J$ & $\frac{C_{T}}{C_{P}}=\frac{\eta_{T}}{\eta_{P}}$ \\
\hline \hline
\end{tabular}

The last row in this table shows that the propeller efficiency $\eta_{\text {prop }}$ can be calculated by using either the ratio of legacy thrust and power coefficients, or the ratio of energy-based normalized thrust and power, as:

$\eta_{\text {prop }}=\frac{C_{T}}{C_{P}} J=\frac{\eta_{T}}{\eta_{P}} J$

The next figure plots the normalized power $\eta_{P}$ and the propeller efficiency $\eta_{\text {prop}}$, against the advance ratio $J$ : 
$P_{N}$ and $\eta v$ v. $J$

@ 4000 rpm / blade pitch=39 deg. @75\%R

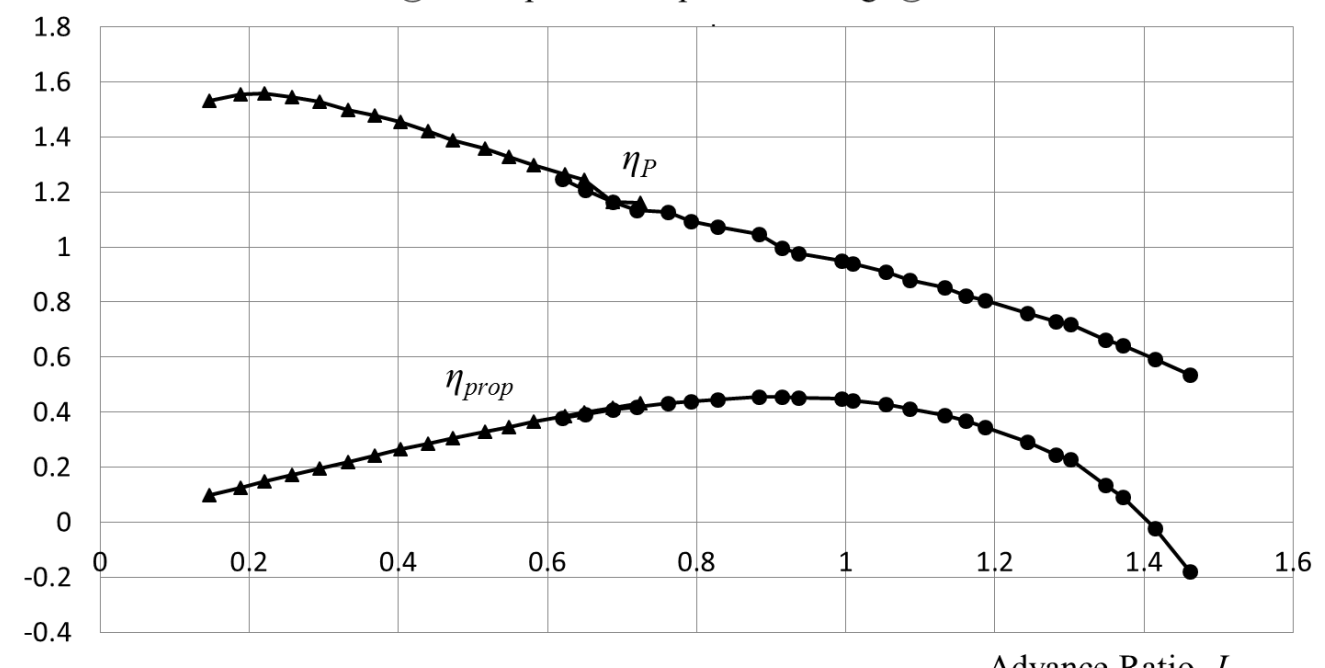

Advance Ratio $J$

Figure 5. Normalized power, and propeller efficiency $\eta_{\text {prop }}$ plotted against $J$ for a $39^{\circ}$ blade pitch.

The translation velocity $v_{\infty}$, the tangential velocity $v_{t i p}$, and the resultant blade velocity $v_{b}$ for a rotating speed of 4,000 rpm are plotted next against advance ratio, $J$ :

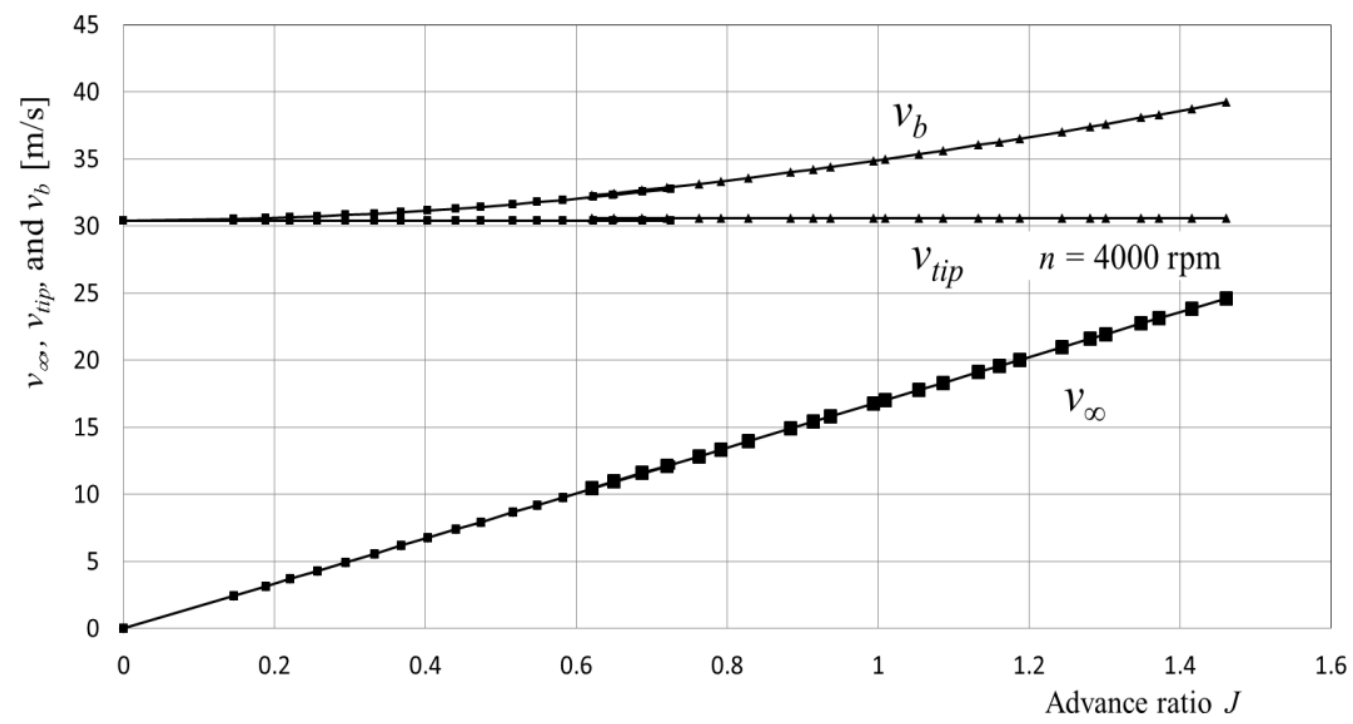

Figure 6. Tip, translation, and blade velocities plotted against the advance ratio, $J$. 
The blade's Reynolds number is calculated using Equation (29) and plotted below against the advance ratio $J$ :

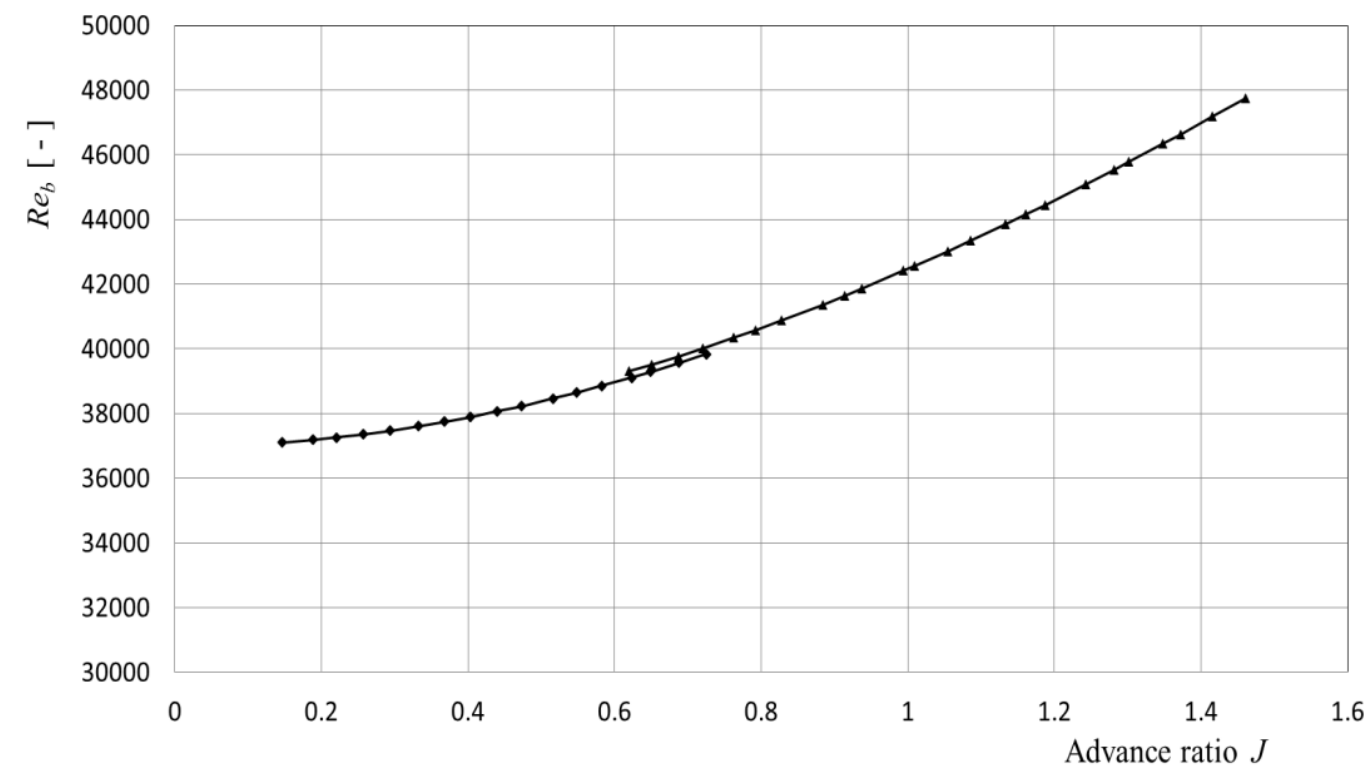

Figure 7. Graph of blade Reynolds number $R e_{b}$ at $0.577 R$ against advance ratio $J$.

It is apparent that a propeller can be designed during its predesign stage in a similar way one would design a wing.

\section{Equilibrium Equations for Propellers and Rotor-driven Vehicles}

Various conceptual and numerical applications are presented next.

\section{The Equilibrium Equation for the Rotor Torque of a Helicopter}

In order to counter the torque imposed by the main rotor of a helicopter, the following equation can be used to predesign its tail rotor:

Helicopter torque $=$ Sideforce by tail rotor $\times$ Moment arm

$$
=K \times\left(\frac{1}{6} v_{\text {tip }}^{2} \rho \eta_{T} S_{b}\right) \times \text { Moment arm }
$$

The factor $K$ multiplying the parentheses containing equation (20) accounts for the effects due to the aerodynamic distortion at the face of the tail rotor due to the main rotor's downwash. 


\section{Calculation of the Blade Area of a Propeller}

In a case of "point design," the total blade area of the propeller powering a Piper Cherokee PA-28-180 (McCormick, 1979) is calculated next. The aircraft flies at a constant true airspeed of $60.25 \mathrm{~m} / \mathrm{s}(197.7 \mathrm{fps})$, a constant altitude of $914.4 \mathrm{~m}$ $(3,000 \mathrm{ft})$, a density $\rho$ of $1.1209 \mathrm{~kg} / \mathrm{m}^{3}(0.002175$ slugs $/ \mathrm{cu} . \mathrm{ft}$.$) and has an$ aerodynamic drag $D$ equal to its thrust $T$ of $1423.4 \mathrm{~N}(145.1 \mathrm{~kg} / 320 \mathrm{lb}$.). Its fixed pitch, single $(N=1)$ two-bladed propeller, has a diameter of $1.88 \mathrm{~m}(6.17 \mathrm{ft}$.), operates at 2,400 rpm $(n=401 / \mathrm{s})$, and at an advance ratio $J\left(=v_{\infty} / n D\right)$ of 0.8 . The normalized thrust of the blade, $\eta_{T}$, is estimated to be 0.7 . The equation for the blade area $S_{b}$ is solved from Equation (41):

$S_{b}=\frac{T}{\frac{1}{2} \rho v_{\infty}^{2}\left[1+\frac{1}{3}\left(\frac{\pi}{J}\right)^{2}\right] \eta_{T}}=\frac{T}{\frac{1}{2} \rho v_{\mathrm{b}}^{2} \eta_{T}}=\frac{T}{Q_{\infty} \eta_{T}}$

The blade area $S_{b}$ of the propeller is $0.162 \mathrm{~m}^{2}(1.75$ sq. $\mathrm{ft}$.$) .$

\section{The Equilibrium Equation of a Propeller-Driven Aircraft}

The drag equation of an aircraft's airframe is a function of the normalized drag $\eta_{D}$ (or its drag coefficient $C_{D}$ if frontal area $S_{f}$ is used as its reference area):

$D=\frac{1}{2} \rho v_{\infty}^{2} \eta_{D} S_{f}$

The above drag equation $D$ of an airplane's airframe translating at a speed $\nu_{\infty}$ is equalized to the thrust equation $T$, Equation (25), generated by a number $N$ of propellers. Simplifying terms $\left(1 / 2 \rho v_{\infty}^{2}\right)$ results in the following equilibrium equation:

$$
\left[1+\frac{1}{3}\left(\frac{\pi}{J}\right)^{2}\right] \eta_{T} S_{b} N=\eta_{D} S_{f}
$$

This equation can be rewritten next by using the equivalent thrust area $f_{T}$ prop of the propellers $\left(=\eta_{T} S_{b}\right)$ and the equivalent drag area $f_{D}$ af of the aircraft's airframe $\left(=\eta_{D} S_{f}\right)$ :

$$
\left[1+\frac{1}{3}\left(\frac{\pi}{J}\right)^{2}\right] f_{T \text { prop }} N=f_{D \text { af }}
$$

The modifier is seen modifying the equivalent thrust area of the propeller to the equivalent drag area of the airframe, or it can be seen acting as a figure of merit: 


$$
\left[1+\frac{1}{3}\left(\frac{\pi}{J}\right)^{2}\right] N=\frac{f_{D a f}}{f_{T \text { prop }}}
$$

This ratio can be fixed by defining the advance ratio $J$ and $N$. Its use is illustrated in the next section.

Next, the normalized drag $\eta_{D}$ (or drag coefficient $C_{D \bullet}$, using Hoerner's nomenclature (Hoerner, 1965) of the same aircraft, a Piper Cherokee (McCormick, 1979 ), is calculated, with a wing area, $S_{p}$, of $14.86 \mathrm{~m}^{2}$ (160 sq. ft.), a total frontal area, $S_{f}$, of $3.56 \mathrm{~m}^{2}$ (36.38 sq. ft.) and same propeller characteristics detailed in the prior subsection. Solving for the normalized drag $\eta_{D}$ in Equation (35) for $N=1$, we obtain:

$$
\eta_{D}=C_{D \bullet}=\left[1+\frac{1}{3}\left(\frac{\pi}{J}\right)^{2}\right] \eta_{T}\left(\frac{s_{b}}{s_{f}}\right)=0.196
$$

Two design parameters can be estimated simultaneously from the above equation: (i) the equivalent drag area, $f_{D a c},\left(=\eta_{D} S_{f}=C_{D} \bullet S_{f}\right)$, which equals $0.69 \mathrm{~m}^{2}$ (7.52 sq.ft.), and (ii) the equivalent thrust area $f_{T}\left(=\eta_{T} \cdot S_{b}\right)$ which equals $0.11 \mathrm{~m}^{2}$ (1.23 sq. ft.).

\section{Conclusion}

The application of the concepts of energy, work and cycle in the field of aerodynamics results in a thrust equation suited to the predesign of propellers and rotors, and can be extended to the calculation of lift, thrust and drag of, say, flapping wings and rotating cylinders in Magnus effect. These equations, which share a common lineage with the ubiquitous lift equation of a translating lifting surface, do not require prior knowledge of the surrounding flow field (as when applying the BEM), and the respective normalized force $\eta_{F}$ can be read on a stand-alone basis (estimating it as a high or low value, relative to a maximum value close to 1 , depending on its Reynolds number), and can be used as a figure of merit to compare the ability to generate aerodynamic forces of a diverse range of systems, regardless of their kinematics.

The empirical nature of the thrust equation acts as a convenient nexus between experiment (i.e., by obtaining, say, the normalized thrust $\eta_{T}$ of coaxial rotors in ground effect or contra-rotating propellers by means of flight testing or wind tunnel testing and matching these values with theoretical tools (i.e., BEM, CFD).

This energy-based perspective may help reduce the existing compartmentalization within aerodynamics (e.g., between rotors and propellers as discussed earlier), facilitate cross-pollination between aerospace, biomechanics, 
International Journal of Aviation, Aeronautics, and Aerospace, Vol. 6 [2019], Iss. 5, Art. 9

and marine propulsion, and contribute to the mathematical groundwork of nascent sciences (e.g., paleoaerodynamics) (Burgers, 2019). 


\section{References}

Anderson, J. D. (2007). Fundamentals of aerodynamics (5th ed.). New York, NY: McGraw-Hill.

Burgers, P. \& Alexander, D. E. (2012). Normalized lift: An energy interpretation of the lift coefficient simplifies comparisons of the lifting ability of rotating and flapping surfaces. PLOS ONE, 7(5), issue 5, e36732. doi:10.1371/journal.pone.0036732

Burgers, P. (2016). Dimensionally and physically proper lift, drag and thrustrelated numbers as figures of merit: Normalized lift, drag and thrust, $\eta_{L}, \eta_{D}$ and $\eta$ T." Journal of Aerospace Engineering. doi:10.1061/(ASCE)AS.19435525.0000689.

Burgers, P. (2019). The replication hypothesis along the take-off run and a system of equilibrium equations at the lift-off of a protobird. Aerospace, 6, 21. doi:10.3390/aerospace6020021.

Gessow, A., \& Myers, G. C. (1952). Aerodynamics of the helicopter (3rd printing). New York, NY: Frederick Ungar.

Halliday, D, \& Resnick R. (1970). Fundamentals of physics. New York, NY: John Wiley.

Harris, F. D. (2011). Autogyros, helicopters and other V/STOL aircraft. NASA SP-2011-215959. Mountain View, CA: Ames Research Center.

Hoerner, S. (1965). Aerodynamic drag. Retrieved from http://www.scirp.org/ (S(351jmbntvnsjt1 aadkposzje))/reference/ReferencesPapers.aspx?Referen ceID $=1882418$

McComick, B. W. (1979). Aerodynamics, aeronautics and flight mechanics. New York, NY: John Wiley.

Moran, M. J., \& Shapiro H. N. (1988). Fundamentals of engineering thermodynamics (1st ed.). New York, NY: John Wiley.

Prandtl, L. (1921). Applications of modern hydrodynamics to aeronautics. NACA TR 116. Retrieved from https://ntrs.nasa.gov/search.jsp? $\mathrm{R}=19930091180$

Roskam, J. (1983). Methods for estimating drag polar of subsonic airplanes (4th printing). Retrieved from https://kissly.net/book/ 8F87AE890B20A80EACF0?utm_source=ps81\&utm_medium=zywewuga cohi.tk\&utm_campaign=fnom \& $\mathrm{x}=303066$

Uhlig, D. V., \& Selig, M. S. (2008). Post stall propeller behavior at low reynolds Numbers. AIAA 2008-407. 46 ${ }^{\text {th }}$ AIAA Aerospace Sciences Meeting and Exhibit. 7-10 January, Reno, Nevada. 


\section{Nomenclature}

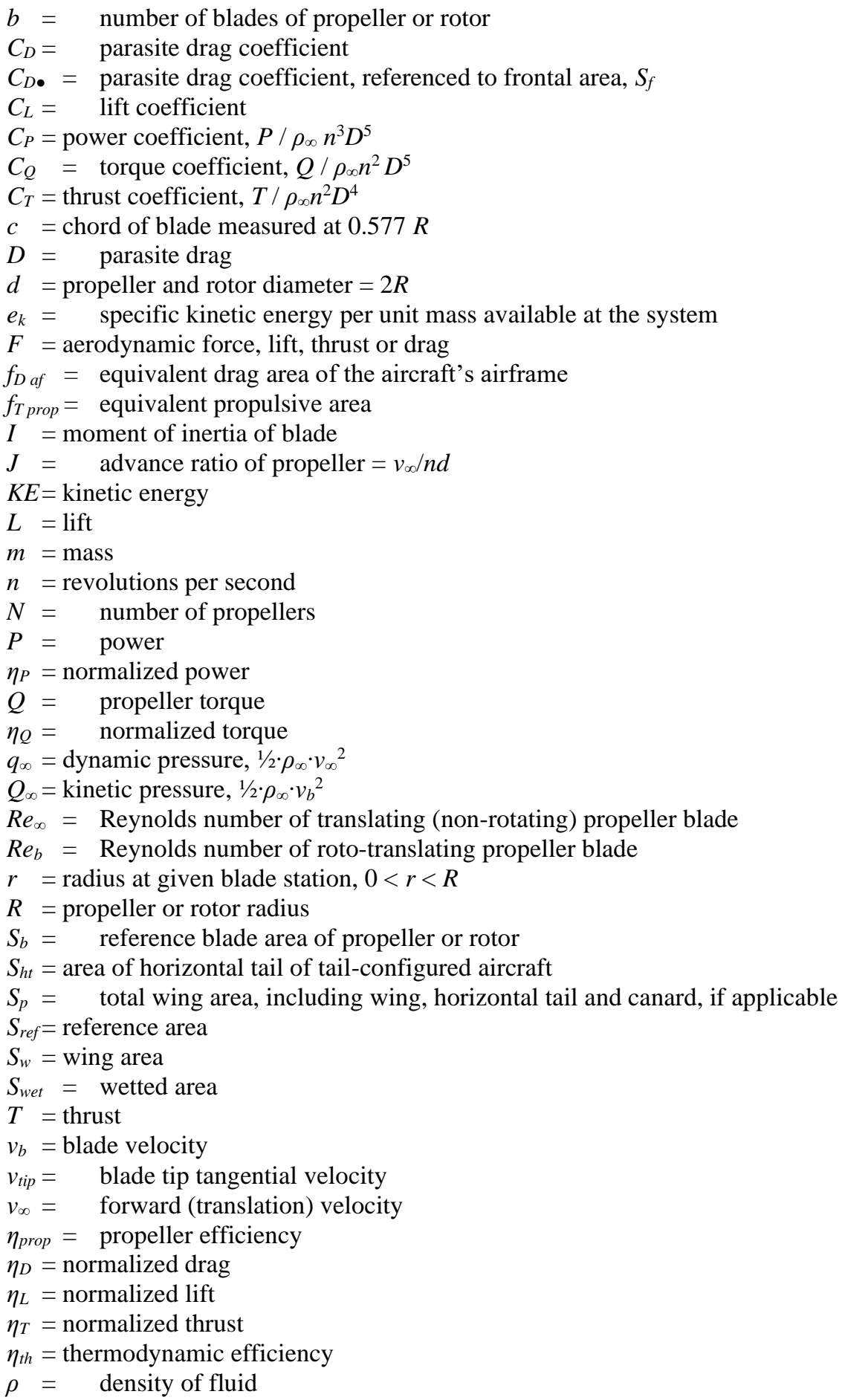


Burgers: An Energy A pproach to a Thrust Equation of Propellers and Rotors

$\omega=$ angular velocity

$\theta=$ angle of attack of blade 